\title{
Responses of Regulator of G Protein Signaling Proteins and Circadian Clock Proteins to Induction of Dedifferentiation in Pancreatic $\beta$-cells
}

Satoshi Okano ( $\sim$ sokano@med.id.yamagata-u.ac.jp )

Yamagata University

Akira Yasui

Tohoku University

Shin-ichiro Kanno

Tohoku University

Kennichi Satoh

Tohoku Medical and Pharmaceutical University

Masahiko Igarashi

Yamagata City Hospital Saiseikan

Osamu Nakajima

Yamagata University

Research Article

Keywords:

Posted Date: March 3rd, 2022

DOI: https://doi.org/10.21203/rs.3.rs-1395694/v1

License: (1) This work is licensed under a Creative Commons Attribution 4.0 International License.

Read Full License 


\section{Abstract}

Regulator of $\mathrm{G}$ protein signaling proteins (RGSs) are involved in regulating $\beta$-cell functions. This study was conducted to examine the cellular responses to the occurrence of $\beta$-cell dedifferentiation in terms of the changes of expression levels of Rgs and clock genes: We used MIN6 cells, for which the cellular contents of zinc were reduced by prolonged treatment of the cells by potassium chloride, in this way inducing the cells to a progenitor-like state. The results indicate that endoplasmic reticulum (ER)-stress markers were up-regulated in the treated cells. All examined RGS were up-regulated in treated cells compared with control cells. RGS2 partly co-localized elF2 a in the treated cells, although no discernable co-localization was observed for RGS4 and 16, suggesting that RGS2 specifically participates in the translational control of protein in response to cellular stresses. Altered mRNA levels of a uniquely specific set of clock genes were observed, suggesting malfunction of the molecular clock in $\beta$-cells. Taken together, $\mathrm{G}$ protein signaling and translation process are probably fine-tuned by RGSs. Thereby $\beta$-cells can cope with stress attributable to the decrease of zinc. The attenuation of circadian rhythm, in addition to some $\mathrm{G}$ protein signaling, can be prerequisites for induction of $\beta$-cell dedifferentiation.

\section{Introduction}

Zinc, an essential nutrient, strongly influences human health: it plays an important role as protein function constituent and regulator in the body. Pancreatic $\beta$-cells are known to contain a large amount of zinc. Zinc is mainly concentrated in the insulin secretory granules, where it plays indispensable roles in insulin crystallization ${ }^{1}$. Consequently, pancreatic $\beta$-cells have high zinc content within cells ${ }^{1}$. Proper zinc homeostasis is a prerequisite for the maintenance of $\beta$-cell function ${ }^{1}$. Constant stimulation of $\beta$ cells by persistent hyperglycemia leads to over-secretion of insulin, which engenders reduction of zinc in $\beta$ cells ${ }^{1}$. Recently, a simple and effective method to reduce cellular zinc using pancreatic $\beta$-cell lines MIN6 was reported, with which the cellular content of zinc is reduced by the appropriate treatment of cells with potassium chloride $(\mathrm{KCl})^{2}$. In this way, MIN6 cells were over-stimulated by the constant depolarization condition of the cells, which mimics hyperglycemia. The experimental systems have revealed some aspects of the influence of zinc depletion ${ }^{2}$. However, the comprehensive cellular responses of $\beta$-cells to the alteration of zinc contents remain unclear.

$\beta$-cells respond to signaling via multiple $G$ protein-coupled receptors (GPCRs) to modify insulin secretion ${ }^{3}$. Regulator of $\mathrm{G}$ protein signaling proteins (RGSs) are involved in the turnoff of $\mathrm{G}$ protein-coupled receptor (GPCR) signaling pathways: they restrict activity of heterotrimeric $G$ protein by acceleration of the inactivation of Ga subunits ${ }^{3}$. Among RGSs, RGS2 is up-regulated in its expression by various stresses ${ }^{4-7}$. Consequently, it is speculated that RGS2 plays modulatory roles in stress responses in addition to its recognized functions in regulating GPCR-mediated signaling. In harmony with that notion, RGS2 is known to be able to reduce global protein synthesis by interaction with the eukaryotic initiation factor $2 \mathrm{~B}$ $(\mathrm{elF} 2 \mathrm{~B})^{8}$. 
Mammalian circadian clocks comprise three interlocked transcriptional-translational feedback loops (TTFLs) at the molecular level. Each loop consists of positive and negative constituents ${ }^{9}$. Although the importance of circadian clock in $\beta$-cell functions has been described in earlier reports ${ }^{10}$, influence of hyperglycemia accompanied by cellular zinc depletion on molecular clock in $\beta$-cells still remains unclear. Results of recent studies suggest that not only $\beta$-cell apoptosis due to oxidative as well as endoplasmic reticulum (ER) stresses but also $\beta$-cell dedifferentiation is involved in the pathogenesis of Type 2 Diabetes ${ }^{11}$. Especially, regarding the molecular mechanisms that engender $\beta$-cell dedifferentiation much more remains to be elucidated. The $\mathrm{KCl}$-treated MIN6 cells can be a beneficial system for investigation of phenomena occurring along with $\beta$-cell dedifferentiation. For detailed elucidation of the characteristics of the dedifferentiation-like state of $\mathrm{KCl}$-treated MIN6 cells, further analysis of their various aspects will be necessary. In this respect, ascertaining how the alteration of proteins involved in molecular clock and RGSs operates in dedifferentiated $\beta$-cells would be revealing, but the answer to this question remains elusive.

To explore the cellular responses of $\beta$-cell to dysregulation of zinc homeostasis as well as the resultant dedifferentiation, we used the zinc-depletion experimental system described above for MIN6 cells treated by $\mathrm{KCl}$. We will report some new features as to the dedifferentiation-like feature of $\mathrm{KCl}$ treated MIN6 cells. Our results suggest that hyperglycemia accompanied by zinc depletion results in distinctive sets of altered expression of Rgs genes and clock genes, which, as consequences might serve important roles in leading to $\beta$-cell dedifferentiation.

\section{Results}

\section{Induction of ER-stress by zinc depletion in MIN6 cells}

Greater knowledge of possible cellular stresses induced by zinc depletion is important to characterize features of $\beta$-cell dedifferentiation in greater detail. For that purpose, first, we examined the cellular stressrelated gene expression. We checked the mRNA level of C/EBP-homologous protein (Chop), heme oxygenase 1 (Ho-1), and thioredoxin-interacting protein (Txnip) genes (Fig.1a). Ho-1 gene expression is induced by various stressors, including oxidative stress ${ }^{12}$. Reportedly, TXNIP is a mediator of oxidative stress induced by glucotoxicity in pancreatic $\beta$-cells ${ }^{11}$. No difference was found between treated and untreated cells in mRNA levels of Ho-1 and Txnip genes (Fig.1a). Inferring that ER-stress would be induced by this treatment due to the hypersecretion of insulin, we checked the expression of the Chop gene, which is induced by stresses including ER-stress ${ }^{13}$. Real-time PCR analysis indicated that the mRNA of Chop was indeed up-regulated (Fig.1, 1.6 fold). These results strongly suggest that oxidative stress was not elicited, but that ER-stress was induced in MIN6 cells by this treatment. We confirmed that mRNA of Chop was prominently elevated by the treatment of thapsigargin $(1 \mu \mathrm{M}, 24 \mathrm{~h})$, which is known to induce ER-stress (Suppl Fig. S1a, 11.1 fold). To verify the occurrence of ER-stress in the KCl-treated MIN6 cells further, we conducted immunostaining analyses for binding immunoglobulin protein (BiP: also known as GRP78]), a molecular chaperone, and activating transcription factor 4 (ATF4), a transcription 
factor. Both proteins play pivotal roles in the unfolded protein response (UPR), which is closely related to ER stress ${ }^{13}$. Results showed that the amount of BiP increased in the ER of the treated cells (Fig.1b). In addition, ATF4 was prominently elevated in the nuclei of the treated cells (Fig.1C), indicating that ERstress was actually induced.

\section{Change in the expression and cellular distribution of RGS proteins by zinc decrease, and their relation to translation repression factor}

We examined mRNA levels of three Rgs genes (Rgs2, Rgs4, and Rgs16), which are expressed abundantly in $\beta$-cells, and which are involved in regulating of proliferation and insulin secretion of pancreatic $\beta$ cells $^{14,15,16}$. Then their subcellular localization was investigated with particular emphasis on the relation with a subunit of elF2 (elF2a), a key player of stress-dependent translational regulation ${ }^{17}$. Rgs2 and Rgs4 genes were prominently up-regulated in treated cells compared with control cells (Fig.2a: Rgs2, 7.0 fold; Rgs4, 5.0 fold). Rgs16 gene was also up-regulated in zinc-reduced MIN6 cells (Fig.2a, 1.9 fold). In the thapsigargin-treated cells, mRNA levels of Rgs 16 genes were more prominently up-regulated compared with control cells than the KCl-treated cases (Suppl Fig. S1: 3.2 fold). Rgs2 gene was also distinctly upregulated in the thapsigargin-treated cells (Suppl Fig. S1b: 3.2 fold). By contrast, mRNA level of Rgs4 was only slightly elevated (Suppl Fig. S1b: 1.1 fold).

Immunostaining experiments revealed that the cellular distribution of elF2a in the $\mathrm{KCl}$-treated cells is drastically changed: cellular foci containing elF2a formed (Fig. 2b), which possibly corresponds to main sites of translational control. RGS2 co-localized in part with the cellular foci containing elF2 $a$ in the treated cells (Fig. 2b), suggesting that RGS2 is also involved in translation control in $\beta$-cells. Although both RGS2 and RGS4 tend to form focal accumulations by KCl treatment in MIN6 cells, no apparent colocalization was found between RGS2 and RGS4 at their respective foci (Fig. 2c). The results strongly suggest that RGS4 is not included in the foci containing elF2 $a$ and RGS2. The results are also consistent

with the results of an earlier report showing that RGS4 is not involved in control of translation ${ }^{8}$. Regarding RGS16, no discernable co-localization with elF2a was observed (Fig. 2d), suggesting that RGS16 is also irrelevant to the regulation of translation. Considered collectively, these results suggest that RGS2 specifically participates in the suppression of translation in response to ER stress in pancreatic $\beta$-cells, at least among the three RGS proteins.

\section{Zinc reduction effects on clock gene expression}

To estimate the effects of the treatment on the molecular circadian clock, we quantified mRNA levels of representative circadian clock genes and performed immunostaining for some key clock proteins. qPCR analyses demonstrated that, in the constituent of the core loop, up-regulation of mRNA levels of Per1 (1.8 fold), Cry 1 (1.3 fold), and down-regulation of Clock ( 0.6 fold) were found in KCl-treated MIN6 cells (Fig.3a). In addition, the mRNA level of Dec1, of which products work as a repressor of CLOCK/BMAL1 heterodimers (hereafter simply referred to as $C / B)^{18}$, was significantly higher than that in control cells (Fig.3a, 1.7 fold). No significant change was observed for Bmal1, Per2 and Cry2 between the respective 
two groups (Fig.3a). CHRONO also represses transcriptional activities of $\mathrm{C} / \mathrm{B}^{19}$. No significant change was observed for Chrono between the two groups (Fig.3a). In the genes of the second loop ${ }^{9}$, mRNA of Rev-erba increased ( 1.6 fold). That of Rory decreased ( 0.5 fold) compared to that of control cells (Fig.3a). Regarding the third loop, up-regulation of E4bp4 (2.0 fold) and down-regulation of $D b p$ ( 0.4 fold) were found in the treated cells (Fig.3a). Therefore, there found an overall tendency that transcription activators are reduced, although repressors of transcription are up-regulated in $\mathrm{KCl}$-treated cells compared with control cells. Furthermore, immunostaining analyses for CLOCK and CRY1, the members of the core loop ${ }^{9}$, were performed to elucidate whether or not changes occur at protein levels. In harmony with the mRNA levels of Clock genes, the amount of CLOCK protein was apparently reduced (Fig.3b). Consistent with the mRNA levels of Cry 1 gene, the protein level of CRY1 in the treated cells was almost the same with those in the controls (Fig.3c).

\section{Dedifferentiation-like features of zinc-depleted MIN6 cells}

An earlier paper reported that the same $\mathrm{KCl}$ treatment as in the present experiment induced loss of identity in $\beta$-cells ${ }^{2}$. In the literature, the dedifferentiation was discussed based on findings of changes of transcription factors at the mRNA level ${ }^{2}$. So far, no report of the relevant literature has described analysis of transcription factors at the protein levels. To proceed further, from analysis of the dedifferentiation of $\beta$-cells, we examined the expression of major transcription factors both at the mRNA and protein levels, particularly the expression of genes for proteins involved in glucose-stimulated insulin secretion (GSIS) and for proteins involved in cellular stresses. We quantified the respective mRNA levels of Insulin2, insulin receptor substrate 2 (Irs2), Glucokinase (Gck), Glut2, and Karyopherin alpha 2 (Kpna2) (Fig.4a). For Kpna2, we examined the gene because we had earlier identified KPNA2 protein as a marker for fully matured $\beta$-cells in an earlier report ${ }^{20}$. No significant difference was found in the expression of mRNA levels of Insulin2 and Irs2 between the two groups (Fig.4a). In contrast, the expression levels of Kpna2 gene were reduced significantly to about $53 \%$ in KCl-treated cells (Fig.4). Expression of Gck, a glucose sensor for $\beta$-cells, decreased to $65 \%$ (Fig.4a). Particularly, the extent of reduction mRNA of Glut2 is extremely large: in the treated cells Glut2 mRNA was reduced to $4 \%$ compared with control cells. We subsequently performed immunostaining analyses for GLUT2 and KPNA2. Consistent with the result of mRNA, the protein level of KPNA2 was reduced (Fig.4b). GLUT2 was reduced drastically in the treated cells compared with control cells (Fig.4c). Then, we examined the expression levels of three transcription factors, NeuroD1, PDX-1, and MafA, which play pivotal roles in the maintenance of cellular functions as mature $\beta$-cells $s^{21}, 22$. The mRNA level of $P d x-1$ was significantly down-regulated to $46 \%$ (Fig.5a). To examine the amounts of PDX-1 protein, immunostaining experiments for PDX-1 were performed. The result demonstrated that the reduction of PDX-1 was observed (Fig.5b). Consequently, both mRNA and protein levels of $P d x-1$ decreased by the reduction of zinc in our experiments. As to NeuroD1, no significant difference was found between the two groups (Fig.5a). which is consistent with the reported result ${ }^{2}$. The mRNA level of MafA decreased severely: The level in the treated cells was decreased to onetenth of that compared with control cells (Fig.5a). We checked the amount of MAFA protein. Consistent 
with the mRNA level, results of immunostaining experiments indicated that MAFA disappeared drastically in the treated cells (Fig.5c).

To briefly check the reduction of cellular zinc in our experiment by the experimental condition presented in the earlier report ${ }^{2}$, we stained MIN6 cells with a zinc-detecting reagent. It is obvious that the levels of staining of the $\mathrm{KCl}$ treated cells were much lower than those of controls, indicating reduction of cellular zinc in the treated cells (Suppl Fig. S2).

To characterize more detailed features of the dedifferentiation-like state in MIN6 cells, we investigate the mRNA level of Aldh1a3, which is known as a marker for dedifferentiation of $\beta$-cells ${ }^{11}$. Results demonstrated that the mRNA level of Aldh1a3 was markedly higher in the treated cells (Fig.6, 3.3-fold). Furthermore, we checked the mRNA levels of hexokinase I ( Hk1) and monocarboxylate carrier 1 (Mct1). Both genes are categorized as "disallowed genes", for which expressions were suppressed in mature $\beta$ cells $^{11}$. The results demonstrated that both mRNA levels were markedly higher than those of control cells (Fig.6, Hk1: 5.3; fold Mct1: 2.4 fold), indicating up-regulation of the expression of these disallowed genes.

\section{Discussion}

Sustained depolarization because of the $\mathrm{KCl}$ treatment must induce insulin hypersecretion. Zinc is cosecreted with insulin from $\beta$-cells ${ }^{1}$. Therefore, insulin hypersecretion might account not only for cellular zinc reduction but also for ER stress because of excess demand of insulin production complementing insulin shortages. In fact, Chop expression was increased to $160 \%$ by the treatment (Fig. 1a). BiP protein accumulated in the ER (Fig. 1b). Also, ATF4 was increased at the protein level in the nucleus (Fig. 1c). These results indicate that ER-stress was indeed induced. No induction of the genes of Ho-1 and Txnip was observed (Fig. 1a), indicating that oxidative stress was not elicited by the treatment. To deepen analyses of cellular stresses, we specifically examined RGS and eukaryotic initiation factor (elF) for their involvement in translational regulation: The purpose was to elucidate the relation between GPCR signaling and stress response of $\beta$-cells caused by zinc depletion. Because elF2B, to which RGS2 can bind $^{8}$, is a regulatory factor for elF $2 a^{17}$, we attempted to co-stain elF2 $a$ and RGSs in MIN6 cells. Results of co-staining showed that RGS2 formed dot-like structures. Some of them co-localized with elF2a under zinc deprivation conditions (Fig. 2b). The co-localization also fits results of increased ATF4 in the treated cells (Fig. 1c) because RGS2 can increase the amount of ATF4 protein in a translation-dependent manner under stress ${ }^{23}$.

The mRNA levels of Rgs2 and Rgs 4 were much higher in the treated cells than in control cells (Fig. 2a); mRNA of Rgs 16 was also elevated in the KCl-treated cells (Fig. 2a), which is consistent with reports describing that the expression of Rgs16 is up-regulated in hyperglycemic conditions in islets of rats ${ }^{16}$ and also in $\beta$-cells from human patients of type 2 diabetes ${ }^{24}$. These results also suggest that in $\beta$-cells various pathways mediated by GPCR can be modulated under hyperglycemia and in consequence the induction of $\beta$-cell dedifferentiation. 
Apparently, RGS4 and 16 are not irrelevant to elF2a (Fig. 2b ,c, d). Collectively, these results suggest strongly that RGS2 is involved in translational control during ER stress in $\beta$-cells. This notion is also supported by the fact that the mRNA level of Rgs2 was up-regulated not only in the KCl-treated cells but also in the thapsigargin-treated MIN6 cells (Suppl. Fig. S1). Our results indicate that stresses associated with zinc depletion significantly affect the expression and the subcellular distribution of various RGSs (Fig. 2). In contrast to the $\mathrm{KCl}$-treated MIN6 cells, the expression level of Rgs4 was scarcely unchanged in the thapsigargin-treated MIN6 cells (Suppl Fig. S1b; 1.1 fold), which may suggest some specific roles of Rgs4 in response to the decrease of zinc due to chronic hyperglycemia in MIN6 cells. The conversion of incretin mediated GPCR-signaling pathways under chronic hyperglycemia in $\beta$-cells was reported recently ${ }^{25}$. It is probable that the modulation of RGS proteins is, at least partially, involved in the reported conversion of signaling pathways.

PDX-1 plays important roles not only in the pancreatic development but also in GSIS in $\beta$-cells ${ }^{21}$. Although it has already been reported that the mRNA level of Pdx-1 was not changed significantly by the same $\mathrm{KCl}$ treatment ${ }^{2}$, our experiments showed that the mRNA level of Pdx-1 was decreased considerably (Fig. 5a). It has been described the decrease of the mRNA of MafA level through treatment (to about $26 \%)^{2}$. In our experiments, the mRNA level of MafA decreased more drastically (to about $11 \%$ ) (Fig. 5a). Although the precise causes of these inconsistencies between the preceding report and our results are currently unknown, the overall tendency of the results shows accordance with earlier reports of the literature ${ }^{2}$. At the protein level, by $\mathrm{KCl}$ treatment MAFA was only slightly observable, and almost completely lost from the nucleus (Fig. 5c). PDX-1 decreased distinctly (Fig. 5b). Expressions of critical genes for GSIS such as Gck and Glut2, which are transcriptional target of PDX-1 ${ }^{21}$, were reduced (Fig. 4a). GLUT2 protein decreased sharply (Fig. 4c). The mRNA levels of $H k 1$ and $M c t 1$, representative $\beta$-celldisallowed genes, and of Aldh1a3 was markedly increased in $\mathrm{KCl}$ treated cells (Fig. 6). These results provide new findings of the characters of dedifferentiation in MIN6 cells. Also, these results strongly suggest that the ability of GSIS decreases with dedifferentiation. To reinforce our notion, we conducted RNA-Seq using the CDNA samples to investigate changes in the transcriptome during the induction of dedifferentiation. The data analysis demonstrated that the $\mathrm{GO}$ function groups of "positive regulation of endothelial cell differentiation " and " endocrine pancreas development " were down-regulated (Suppl Fig. S3). In addition, "response to glucose" and "positive regulation of insulin secretion" were also lowered in the treated cells (Suppl Fig. S3). These results further support the above-mentioned interpretations.

We previously pointed out that, based on experiments using mice, chronic hyperglycemia might be involved in decreased KPNA2 expression, which can account for $\beta$-cell dysfunction ${ }^{20}$. The mRNA level of Kpna2 was decreased by the $\mathrm{KCl}$ treatment (Fig. 4a). The mRNA level of Kpna2 was much lower in thapsigargin-treated cells than in control cells (Suppl Fig. S1c). Taken together, these results suggest that the expression level of KPNA2 is reduced by cellular stresses in $\beta$-cells.

Regarding the response of clock genes in the $\mathrm{KCl}$-treated MIN6 cells, the expression of E4bp4, a repressor of the second-loop of $\mathrm{TTFL}^{9}$, increased and that of $\mathrm{Dbp}$, a transcriptional activator, decreased by $\mathrm{KCl}$ 
treatment (Fig. 3a). These features of E4bp4 and Dbp is consistent with the literature ${ }^{26}$ for ER-stress induced in MIN6 treated by thapsigargin, suggesting that the effect of ER-stress is partially involved in the changes of clock genes in the $\mathrm{KCl}$-treated cells. Although our results showed that zinc depletion altered mRNA levels of a uniquely specific set of clock genes (Fig. 3a), the feature of alteration was essentially different from the case previously reported in thapsigargin-treated MIN6 cells ${ }^{26}$. For instance, in thapsigargin-treated MIN6 cells, genes of Clock and Bmal1 are up-regulated, while mRNA level of Reverba is not affected ${ }^{26}$. In all three loops of TTFL, a decrease was found in positive transcriptional activators (Clock, Dbp, Rory) and an increase in negative transcriptional repressors (Cry1, E4bp4, Dec1, Rev-erba) (Fig. 3a). Judging from the results, it is conceivable that for all the loops that constitute TTFLs, the amplitude of rhythm was damped and attenuated. Therefore, the attenuation of molecular circadian rhythm is invariably induced by stress because of the zinc decrease. In harmony with the idea, the mammalian circadian clock development strongly couples with the cellular status of differentiation ${ }^{27}$. As described in the literature ${ }^{28}, \mathrm{CRY}$ s can be susceptible to zinc; its repressor activity to C/B can be altered by zinc. Results of the present study demonstrated that Cry 2 was unchanged and that $C r y 1$ was upregulated slightly in zinc-reduced MIN6 cells (Fig. 3a). No prominent change in the cellular distribution of CRY1 protein was observed (Fig. $3 \mathrm{c}$ ). Thus, the results show that at least CRY1 proteins can continue to be present in situations of cellular zinc reduction and that they presumably continue to be involved in the signaling of the change in the concentration of cellular zinc: CRY proteins may serve as one of the mediators of the information of zinc depletion in $\beta$-cells. Thereby $\beta$-cells can cope with various stresses accompanied by zinc depletion. The idea is in harmony with our preceding result that zinc-insensitive CRY1 overexpressing mice display diabetes with $\beta$-cell dysfunction ${ }^{10}$.

In summary, our results suggest that cellular stresses caused by zinc depletion induce not only the altered expression levels of Rgs genes, but also the distributional changes of their proteins. Speculation that these consequences must be responsible for induction of $\beta$-cell dysfunction as well as of $\beta$-cell dedifferentiation is tempting. Our results strongly suggest that crosstalk exists between the molecular processes of the loss of $\beta$-cell identity and the attenuation of the molecular clock in $\beta$-cells. These fundamental results can be clues leading to inventions supporting pharmaceutical therapy of diabetes mellitus.

\section{Methods}

\section{Cell culture}

Mouse insulinoma MIN6 cells were a kind gift from Prof. Jun-ichi Miyazaki, Osaka University. MIN6 cells were cultured and treated with $40 \mathrm{mM} \mathrm{KCl}$ for $24 \mathrm{hr}$ similarly to a description presented in the literature ${ }^{2}$.

\section{Immunofluorescence Microscopy}


MIN6 cells were fixed with methanol-acetone solution and were immunolabelled mainly as described in an earlier report ${ }^{29}$. Relevant primary and secondary antibodies are listed in Supporting Information. Nuclear DNA was counterstained with 4',6'-diamidino-2-phenylindole (DAPI; Fujifilm Wako Pure Chemical Corp.). The cell samples were mounted in drops of fluorescence mounting medium (Dako Omnis; Agilent Technologies, Inc.). Coverslips were added. Fluorescence microscopic images were obtained using a fluorescence microscope (BZ-X700; Keyence Corp.).

\section{Quantification Of Mrna By Real-time Pcr}

The cDNA samples of MIN6 cells used for experiments were acquired as described in our earlier report ${ }^{30}$. Real-time PCR analyses were conducted as described in an earlier report ${ }^{30}$. The measured expression levels are expressed as relative values with respect to Actin levels. Primer sets for $P d x-1$, Insulin2, Gck, Glut2, Per1, Per2, Rev-erb $a$, and Dbp were described in an earlier report ${ }^{30}$, as were primer sets for Cry 1 and $B m a / 1$, primer sets for $R g s 16^{31}$, and the primer set for $K p n a 2^{15}$. Other primer sets are listed in Supporting Information.

\section{Statistical Analysis}

For each experiment, a $t$-test was used to compare the mean values. Differences between means were inferred as significant for $P<0.05$. Data are presented as mean $\pm \mathrm{SE}$.

\section{Declarations}

\section{Author contributions}

S-I.K., A.Y., S.K. and N.O. technically supported the experiments. S.O. S.K. and M.I. participated in the interpretation of the results. S.O. designed the research and performed all experiments, analyze the data, as well as integrate the interpretation of the results. S.O. wrote the manuscript.

\section{Data availability}

The data that supports the findings of this study are available from the corresponding author on reasonable request.

\section{Competing interests}

The authors declare no competing interests.

\section{Acknowledgments}

S.O. would like to thank Profs. Maureen Gannon (Vanderbilt Univ.) and Emeritus Prof. Kiyoshi Hayasaka (Yamagata Univ.) for discussions and warm encouragement. This work was supported by JSPS KAKENHI (Grant No.: $15 \mathrm{~K} 08417$ (S.O.) and 19K07498 (S.O.)) and by the Joint Research Program of Joint 
Usage/Research Center at the Institute of Development, Aging and Cancer (IDAC), Tohoku University (Grant Nos.: 30 [2018] (S.0.), 8 [2019] (S.0.), 10 [2020] (S.O.) and 38 [2021] (S.0.)).

\section{References}

1. Chabosseau, P. \& Rutter, G. A. Zinc and diabetes. Arch Biochem Biophys 611, 79-85 (2016).

2. Lawson, R. W. Maret \& Hogstrand, C. Prolonged stimulation of insulin release from MIN6 cells causes zinc depletion and loss of beta-cell markers. J Trace Elem Med Biol 49, 51-59 (2018).

3. Persaud, S. J. Islet G-protein coupled receptors: therapeutic potential for diabetes. Curr Opin Pharmaco/37,24-28 (2017).

4. Ota, A. Sawai, M. \& Sakurai, H. Stress-induced transcription of regulator of G protein signaling 2 (RGS2) by heat shock transcription factor HSF1. Biochimie95, 1432-1436 (2013).

5. Song, L. \& Jope, R. S. Cellular stress increases RGS2 mRNA and decreases RGS4 mRNA levels in SHSY5Y cells. Lett 402, 205-209 (2006).

6. Zmijewski, J. W. et al. Oxidative stress and heat shock stimulate RGS2 expression in 1321N1 astrocytoma cells. Arch, Biochem Biophys392, 192-196 (2001).

7. Endale, M. et al. Ischemia induces regulator of $G$ protein signaling 2 (RGS2) protein upregulation and enhances apoptosis in astrocytes. Am J Physiol Cell Physio/298, C611-C623 (2010).

8. Nguyen, C. H. et al. Translational control by RGS2. Cell Bio/186, 755-765 (2009).

9. de Assis L. V. M. \& Oster, H. The circadian clock and metabolic homeostasis: entangled networks. Cell Mol Life Sc178, 4563-4587 (2021).

10. Okano, S. Unique Aspects of Cryptochrome in Chronobiology and Metabolism, Pancreatic beta-Cell Dysfunction, and Regeneration: Research into Cysteine414-Alanine Mutant CRY1.J Diabetes Res2016,3459246 (2016).

11. Christensen, A. A. \& Gannon, M. The Beta Cell in Type 2 Diabetes. Curr Diab Rep19, 81 (2019).

12. Loboda, A. et al. Role of Nrf2/HO-1 system in development, oxidative stress response and diseases: an evolutionarily conserved mechanism. Cell Mol Life Sci 73, 3221-3247 (2016).

13. Almanza, A. et al.Endoplasmic reticulum stress signalling - from basic mechanisms to clinical applications. FEBS $\mathbf{2} \mathbf{2 8 6 , 2 4 1 - 2 7 8 ~ ( 2 0 1 9 ) . ~}$

14. Klinger, S.et al. Increasing GLP-1-induced beta-cell proliferation by silencing the negative regulators of signaling cAMP response element modulator-alpha and DUSP14. Diabetes57 584-593 (2008).

15. Ruiz de Azua, I.et al.RGS4 is a negative regulator of insulin release from pancreatic beta-cells in vitro and in vivo. Proc Natl Acad Sci U S A107, 7999-8004 (2010).

16. Vivot, K.et al. The regulator of G-protein signaling RGS16 promotes insulin secretion and $\beta$-cell proliferation in rodent and human islets. Mol Metab5, 988-996 (2016).

17. Donnelly, N. Gorman, A. M. Gupta, S. \&Samali, A. The elF2a kinases: their structures and functions. Cell Mol Life Sci 70, 3493-3511 (2013). 
18. Honma, S. et al. Dec1 and Dec2 are regulators of the mammalian molecular clock. Nature419, 841844 (2002).

19. Goriki, A. et al. A novel protein, CHRONO, functions as a core component of the mammalian circadian clock. PLoS Biology12, e1001839 (2014).

20. Okano, S. et al. Karyopherin alpha 2-expressing pancreatic duct glands and intra-islet ducts in aged diabetic C414A-mutant-CRY1 transgenic mice. J Diabetes Res2019, 7234549 (2019).

21. Kume S. The molecular basis and prospects in pancreatic development.Dev Growth Differ47,367-374 (2005).

22. Kaneto $\mathrm{H} \&$ Matsuoka $\mathrm{T} A$. Role of pancreatic transcription factors in maintenance of mature $\beta$-cell function. Int J Mol Sci16, 6281-6297 (2015).

23. Wang, C. J. \& Chidiac, P. RGS2 promotes the translation of stress-associated proteins ATF4 and CHOP via its elF2B-inhibitory domain. Cell Signa/59, 163-170 (2019).

24. Avrahami, D. et al. Single-cell transcriptomics of human islet ontogeny defines the molecular basis of $\beta$-cell dedifferentiation in T2D. Mol Metab42, 101057 (2020).

25. Oduori, O. S. et al. Gs/Gq signaling switch in beta cells defines incretin effectiveness in diabetes. $J$ Clin Invest130, 6639-6655 (2020).

26. Ohta, Y. et al. Clock Gene Dysregulation Induced by Chronic ER Stress Disrupts $\beta$-cell Function. EBioMedicine2017,146-156 (2017).

27. Yagita, K. et al. Development of the circadian oscillator during differentiation of mouse embryonic stem cells in vitro. Proc Natl Acad Sci U S A107, 3846-3851 (2010).

28. Schmalen, I. et al. Interaction of circadian clock proteins CRY1 and PER2 is modulated by zinc binding and disulfide bond formation. Cel/157, 1203-1215 (2014).

29. Okano, S. et al. Spatial and temporal cellular responses to single-strand breaks in human cells. Mol Cell Bio/23, 3974-3981 (2003).

30. Okano, S. et al.Characterization of age-associated alterations of islet function and structure in diabetic mutant cryptochrome 1 transgenic mice. J Diabetes Investig428-435 (2013).

31. Okano, S. Yasui, A. Hayasaka, K. \& Nakajima, O. Unique food-entrained circadian rhythm in cysteine414-alanine mutant mCRY1 transgenic mice. Sleep Biol Rhythms14, 261-269 (2016).

\section{Figures}


a

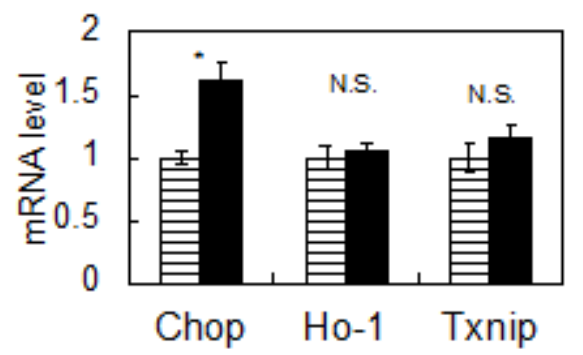

b

$\mathrm{BiP}$

DAPI

Control
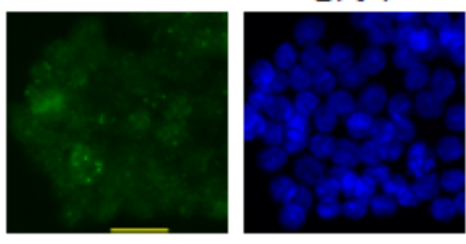

$\mathrm{KCl}$
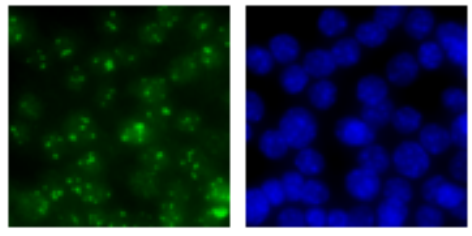

C

ATF4

DAPI

Control
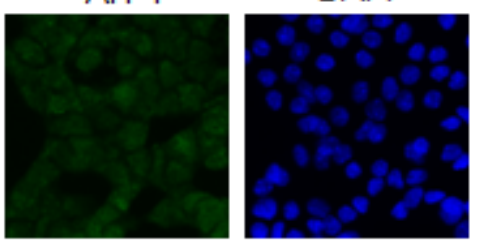

$\mathrm{KCl}$
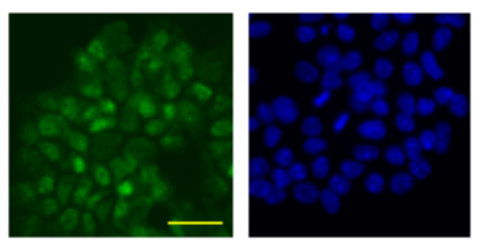

\section{Figure 1}

mRNA and protein expression of genes involved in cellular stresses (Chop, Ho-1, and Txnip) in MIN6 cells.amRNA expression of Chop, Ho-1, and Txnip. In each representation, the control cell value was set to 1. Each value (horizontally lined bars, control; black bars, KCl-treated) represents mean $\pm \mathrm{SE}$ (control, $n=7$; treated, $n=6)$. ( ${ }^{\star} P<0.01, t$ test). N.S. signifies that no significant difference was found between the two groups. Protein expression of BiP (b) and ATF4 (c)in MIN6 cells. Cells were fixed and stained with antiBiP antibody and anti-ATF4 antibody respectively. BiP (b) and ATF4 (c)appear green (left panels). DNA was stained with DAPI (right panels). Bar: $20 \mu \mathrm{m}$ 


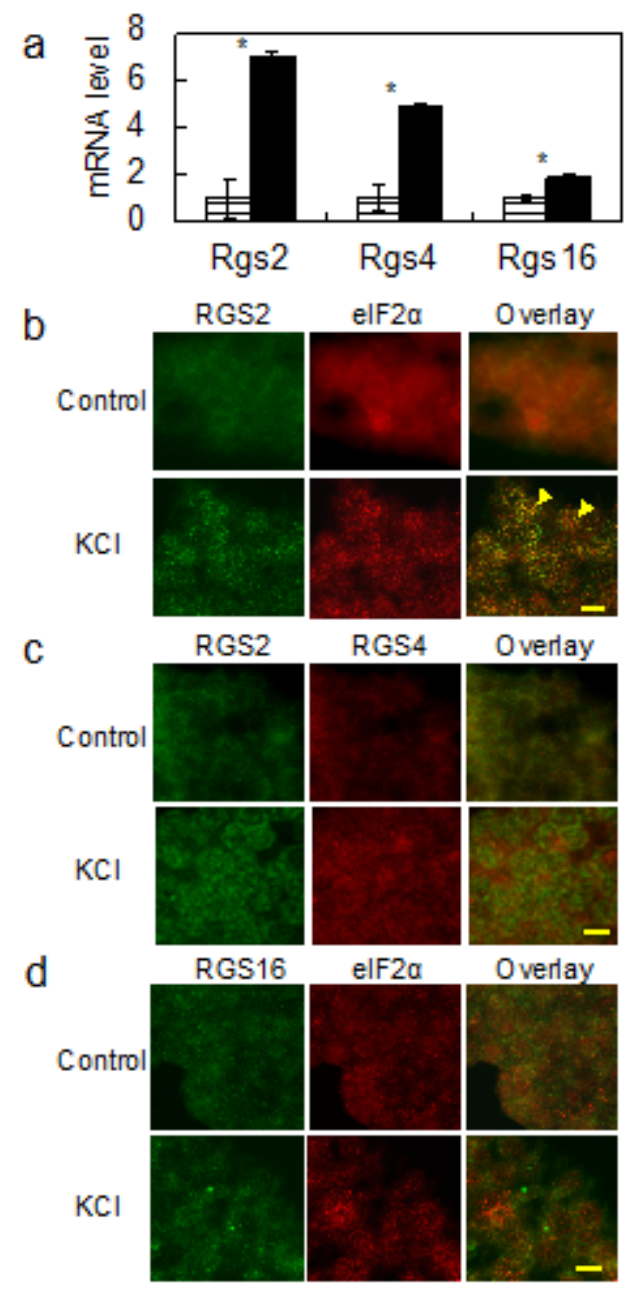

\section{Figure 2}

mRNA and protein expression of Rgs genes (Rgs2, Rgs4, and Rgs16) in MIN6 cells. amRNA expression of $R g s 2, R g s 4$, and $R g s 16$. In each representation, the controls cell value was set to 1 . Each value (horizontally lined bars, control; black bars, $\mathrm{KCl}$-treated) represents mean $\pm \mathrm{SE}$ (control, $n=7$; treated, $n=6$ ). ( ${ }^{\star} P<0.001, t$ test). b Fluorescent micrographs of MIN6 cells acquired by double immunolabeling for RGS2 and elF2a. Cells were fixed and stained with anti-RGS2 antibody (green, left panels), and anti- elF2a antibody (red, middle panels). Both fluorescent images were merged (right panels). Co-localization of both RGS2 and elF2a appears yellow in the overlay. Yellow triangles indicate co-localization. Bar: $10 \mu \mathrm{m}$. c Fluorescent micrographs of MIN6 cells acquired by double immunolabeling for RGS2 and RGS4. Cells were fixed and stained with anti-RGS2 antibody (green, left panels), and anti-RGS4 antibody (red, middle panels). Both fluorescent images were merged (right panels). No specific co-localization of RGS2 and RGS4 in the treated cells was observed in the overlay. Bar: $10 \mu \mathrm{m}$. D. Fluorescent micrographs of MIN6 cells acquired by double immunolabeling for RGS16 and elF2a. Cells were fixed and stained with antiRGS16 antibody (green, left panels), and anti- elF2 $a$ antibody (red, middle panels). Both fluorescent images were merged (right panels). No specific co-localization of both RGS16 and elF2a in the treated cells was observed in the overlay. Bar: $10 \mu \mathrm{m}$. 
a

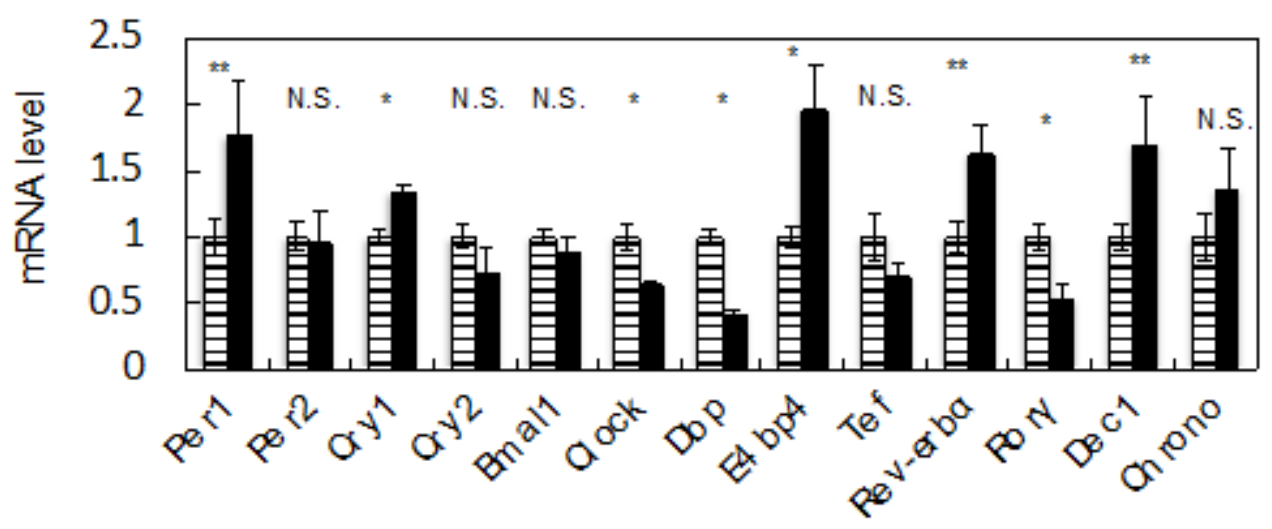

b

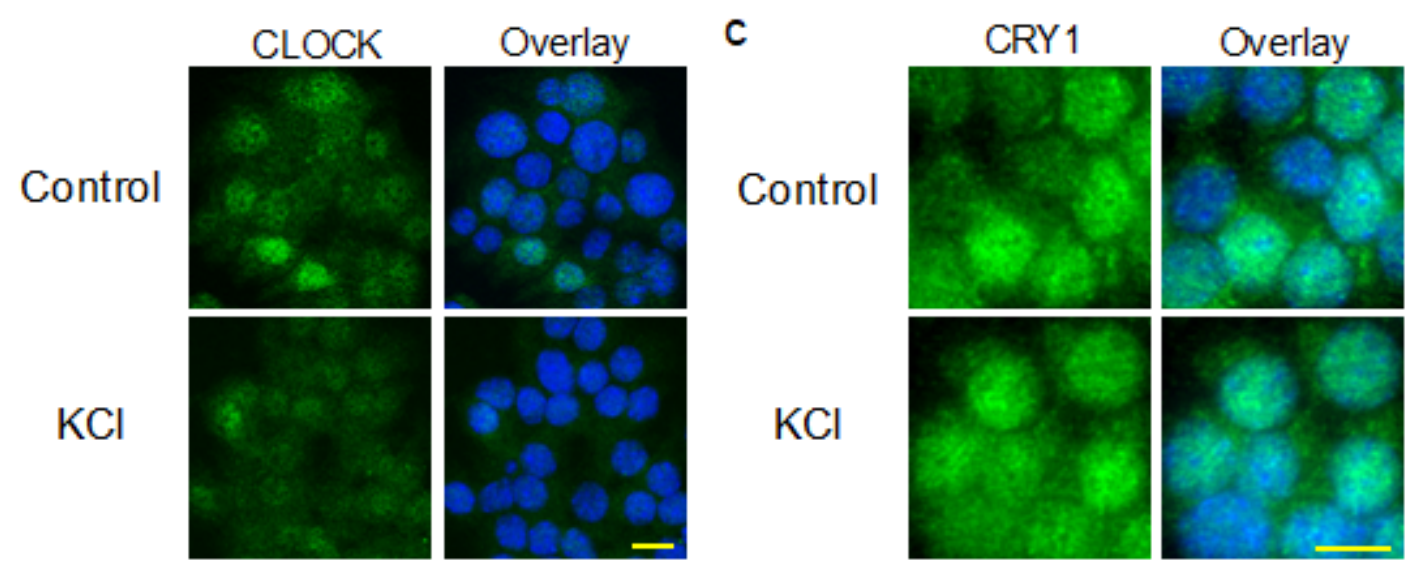

Figure 3

mRNA and protein expression of circadian clock genes in MIN6 cells. a mRNA expression of Per1, Per2, Cry1, Cry2, Bmal1, Clock, Dbp, E4bp4, Tef, Rev-erba, Rory, Dec1, and Chrono. In each representation, the value for the controls cells was set to 1 . Each value (horizontally lined bars, control; black bars, $\mathrm{KCl}$ treated) represents mean $\pm \mathrm{SE}$ (control, $n=7$; treated, $n=6)\left({ }^{\star} P<0.01, t\right.$ test). N.S. signifies that no significant difference was found between the two groups. Increase of mRNA levels of Per1, Cry 1, Dec1, Rev-erba, and E4bp4, and decrease of Clock,Rory and Dbp were observed in KCl-treated MIN6 cells. No significant change was found for Bmal1, Per2, Cry2, or Chrono between the two groups. Protein expression of CLOCK (b)and CRY1 (c) in MIN6 cells. Fluorescent micrographs of MIN6 cells obtained by immunolabeling for CLOCK (b)and CRY1 (c). DNA was stained with DAPI and superimposed onto the fluorescent image (right panels). bBar: $20 \mu \mathrm{m} . c B a r: 10 \mu \mathrm{m}$. 
a

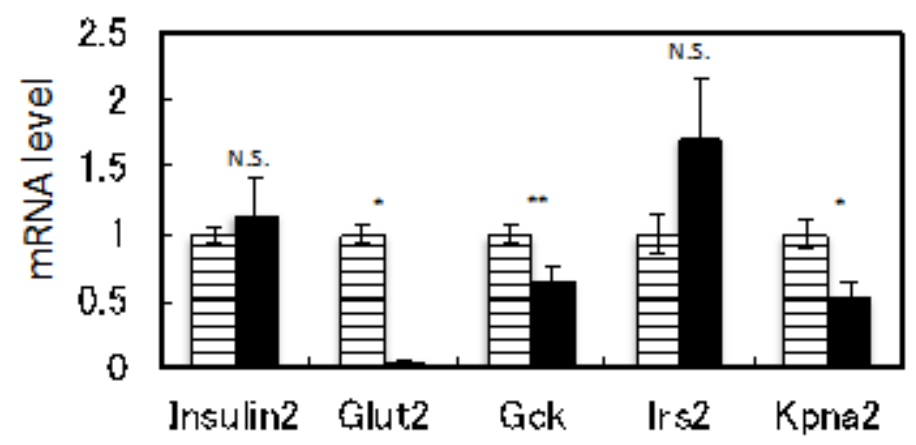

b

C

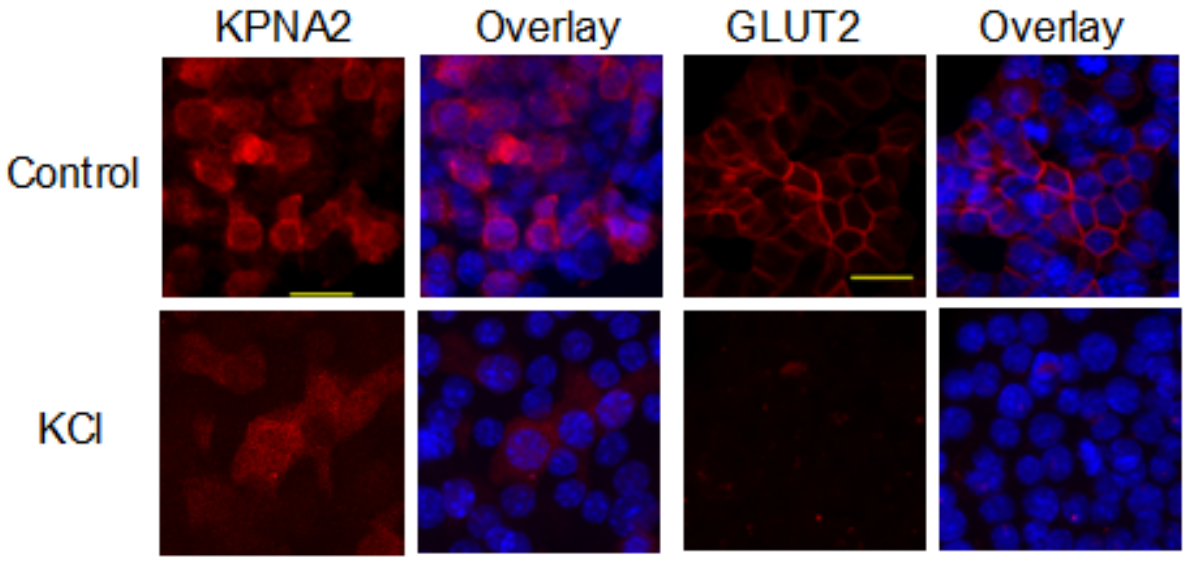

\section{Figure 4}

mRNA and protein expression of $\beta$-cell expressing genes in MIN6 cells. a mRNA expression of genes (Insulin2, Glut2, Gck, Irs2 and Kpna2). In each representation,the value for the control cells was set to 1. Each value (horizontally lined bars, control; black bars, $\mathrm{KCl}$-treated) represents mean $\pm \mathrm{SE}$ (control: $n=7$, treated: $n=6) .\left({ }^{\star} * P<0.05,{ }^{\star} P<0.01,{ }^{\star} P<0.001, t\right.$ test). N.S. signifies that no significant difference exists between the two groups.

b Fluorescent micrographs of MIN6 cells obtained by immunolabeling for KPNA2 andGLUT2. KPNA2, GLUT2 and MafA appear as red (left panels). DNA was stained with DAPI and superimposed onto the fluorescent image (right panels). Bar: $20 \mu \mathrm{m}$. 
a

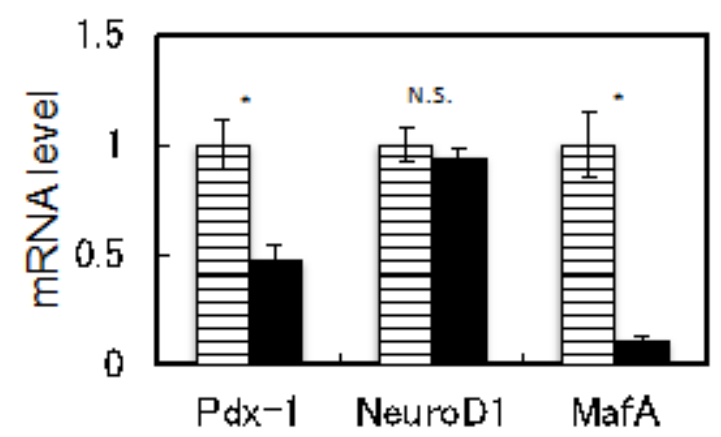

b

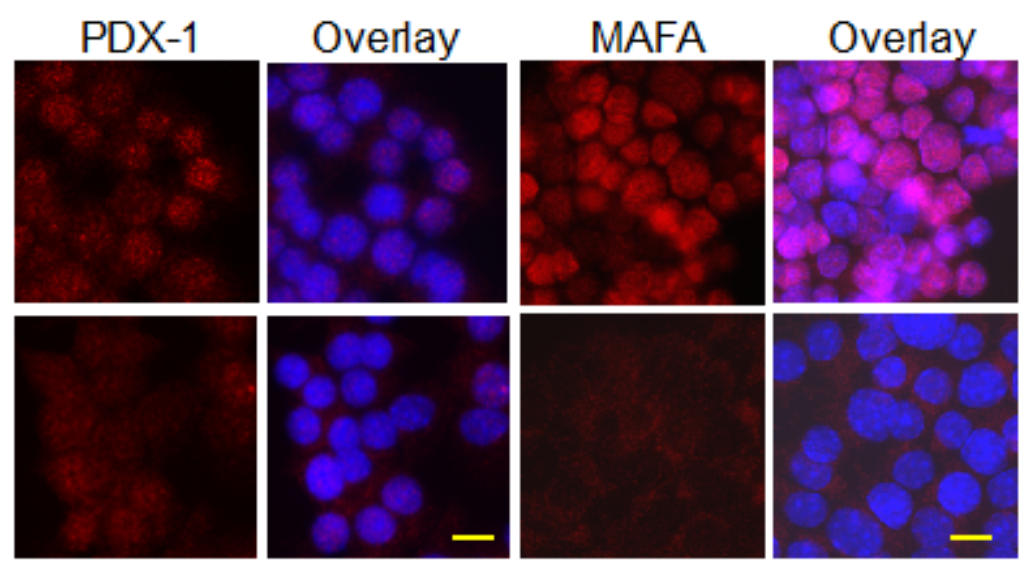

Figure 5

mRNA and protein expression of transcription factors $(P d x-1$, NeuroD1, and MafA) in MIN6 cells. a mRNA expression of genes ( $P d x-1, N e u r o D 1$, and MafA). In each representation, the value for the control cells was set to 1. Each value (horizontally lined bars, control; black bars, $\mathrm{KCl}$-treated) represents mean $\pm \mathrm{SE}$ (control: $n=7$, treated: $n=6)$. ( ${ }^{\star} P<0.01, t$ test). N.S. signifies that no significant difference exists between the two groups.b Fluorescent micrographs of MIN6 cells obtained by immunolabeling for KPNA2 (b)and MAFA (c). KPNA2and MAFA appear as red (left panels). DNA was stained with DAPI and superimposed onto the fluorescent image (right panels). Bar: $10 \mu \mathrm{m}$.

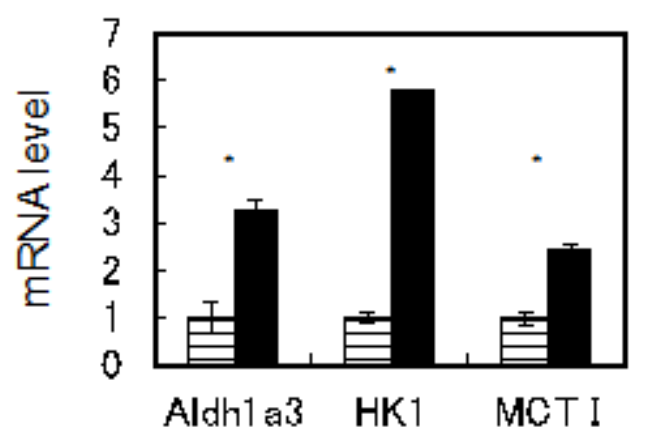

Figure 6 
mRNA and protein expression of $\beta$-cell disallowed genes (Aldh1a3, Hk1, and Mct1). In each representation, the value for the control cells was set to 1 . Each value (horizontally lined bars, control; black bars, $\mathrm{KCl}$ treated) represents mean \pm SE (control: $n=7$, treated: $n=6)$. ( ${ }^{\star} P<0.01, t$ test).

\section{Supplementary Files}

This is a list of supplementary files associated with this preprint. Click to download.

- 225SRSupplementaryMaterials.docx 